\title{
Dual Band Magnonic Crystals: Model System and Basic Spin Wave Dynamics
}

\author{
Federico Montoncello and Loris Giovannini \\ Dipartimento di Fisica e Scienze della Terra, Università degli Studi di Ferrara, Via G. Saragat 1, 44121 Ferrara, Italy \\ Correspondence should be addressed to Federico Montoncello; montoncello@fe.infn.it
}

Received 3 December 2015; Revised 13 April 2016; Accepted 23 May 2016

Academic Editor: Charles C. Sorrell

Copyright (C) 2016 F. Montoncello and L. Giovannini. This is an open access article distributed under the Creative Commons Attribution License, which permits unrestricted use, distribution, and reproduction in any medium, provided the original work is properly cited.

\begin{abstract}
We investigate a special design of two-dimensional magnonic crystal, consisting of two superimposed lattices with different lattice constants, such that spin waves (SWs) can propagate either in one or the other sublattice, depending on which of the two frequency bands they belong to. The SW bands are separated by a very large bandgap (in our model system, $6 \mathrm{GHz}$ ), easily tunable by changing the direction of an applied magnetic field, and the overlap of their spatial distribution, for any frequency of their bands, is always negligible. These properties make the designed system an ideal test system for a magnonic dual band waveguide, where the simultaneous excitation and subsequent propagation of two independent SW signals are allowed, with no mutual interference.
\end{abstract}

\section{Introduction}

In recent years the field of magnonics has received considerable attention and has developed very fast: the idea of manipulating spin wave (SW) propagation, through its Bragg diffraction in artificial lattices in which magnetic properties are periodically varied (magnonic crystals, MCs), has been extensively investigated in numerous systems, both theoretically and experimentally, with numerous applications $[1,2]$. Depending on geometry, material, magnetization, and applied field, collective SWs in MCs can be controlled at will for specific purposes and applications [3-8]. Versatile devices have been proposed, in which SWs can propagate at a tunable group speed, which can turn from waveguides to memories and vice-versa under the action of some external field [916] and logic gates where the output signal is determined by specific interactions between input propagating SWs [1719]. The use of SWs as information carriers is particularly interesting in the context of limiting energy dissipation, with reference to ohmic losses, since no direct electron motion is involved [20,21], and hence magnonic devices are considered key ingredients in the context of enhancing the device efficiency, even when integrated with the traditional ones.
In this paper we investigate a special two-dimensional (2D) MC, designed in such a way that SWs can propagate in distinct regions of it, depending on the frequency band they belong to. We characterize the two spin wave types, by determining the two localization areas and the extension of the corresponding bands.

\section{Calculation Methods and System Design}

We study the system within the micromagnetic framework, computing the equilibrium magnetization by the software OOMMF [22] and the spin wave dynamics by the dynamical matrix method (DMM) [23]. The equilibrium maps provided by OOMMF are the input of the DMM software, which calculates the normal mode frequencies and spatial profiles within a Hamiltonian framework, after computing a matrix, on which an eigenvalue/eigenvector problem is solved for any wavevector value and direction. Eigenvalues and eigenvectors provide spin wave frequency and spatial profile, respectively. We used the following magnetic parameters: saturation magnetization $M_{s}=800 \mathrm{kA} / \mathrm{m}$, exchange stiffness constant $A=$ $1.3 \times 10^{-11} \mathrm{~J} / \mathrm{m}$, and gyromagnetic ratio $\gamma=185 \mathrm{rad} \mathrm{GHz} / \mathrm{T}$. An external magnetic field of constant magnitude $B_{0}=0.1 \mathrm{~T}$ is applied, at different directions, as shown in Figure 1. 


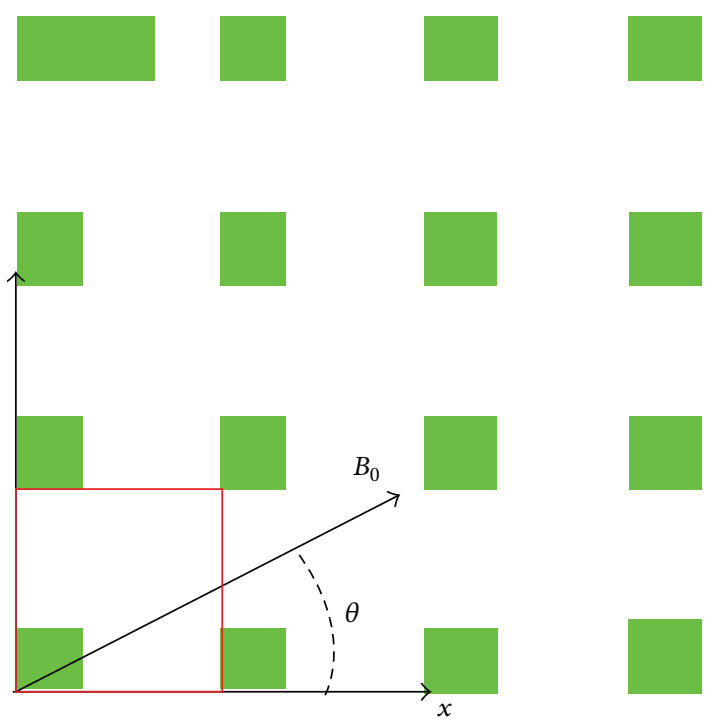

Figure 1: Primitive cell of the 2D magnonic crystal used for calculations. The angle $\theta$ is the direction at which the external magnetic field $B_{0}$ is applied. Distance between the small squares is $a_{1}=15 \mathrm{~nm}$. This cell is unitary only for the secondary sublattice $\left(a_{2}=4 a_{1}\right)$. The red line square is the ideal unitary primitive cell of the primary sublattice (which was however not directly used in the calculations).

Due to the long-range nature of dipolar interactions, it is not trivial to find out a $2 \mathrm{D}$ periodic magnetic system, in which the magnetization dynamics involve two specific and distinct regions of it for all modes in two complete frequency bands, and, at the same time, the two bands are enough separated in frequency. To this purpose, the basic idea is using shape anisotropy to differentiate in energy the two sublattices within the primitive cell, and this is attained with the following MC design (Figure 1): a main (primary) square sublattice, with a short lattice constant $\left(a_{1}=15 \mathrm{~nm}\right)$, is made of cubes with a side of $5 \mathrm{~nm}$, that is slightly lower than the exchange correlation length $\left(\lambda_{\mathrm{ex}}=5.5 \mathrm{~nm}\right.$, in our case), and exactly corresponding to the micromagnetic elemental cell. A secondary square sublattice, with a long lattice constant $\left(a_{2}=60 \mathrm{~nm}\right)$, is made of larger prism particles, with identical thickness and width, but with a double length: $10 \times 5 \times 5 \mathrm{~nm}^{3}$ (hence consisting of two micromagnetic cells). In this way, the dipolar energy in smaller particles is larger than that in bigger particles, and this is a condition necessary to both increase the frequency of the modes localized in larger particles and determine a fast decay of the mode oscillation outside that region. Actually, the two lattices that we have chosen are not geometrically disjoint, but an element of the primary sublattice lays always over the left part of any element of the secondary sublattice. However, this is not altering the overall purposes and results, because the intersection area is always not involved by dynamic magnetization of the primary lattice in no cases: the two lattices can be seen as always dynamically disjoint.

We would like to comment our choice in the perspective of an experimental investigation. Apparently, due to the reduced dimensions of the nanoparticles, our MC system might look rather academic: as already remarked, the design of the present $\mathrm{MC}$ is intended to test the feasibility of dual propagation of independent signals within the same medium. However, the lowest limit of current technology (either by self-assembly techniques or by bioengineered crystallization) is a crystal dimension within $10 \div 5 \mathrm{~nm}$, that is, rather close to our choice, $5 \mathrm{~nm}$ [24-26]. Moreover, in recent past other systems based on very small particles were investigated ([27] and references therein). At present, the investigated systems, usually composed of bigger elements, sometimes with different magnetic materials, allow spin waves with different localization features, which however belong to the same frequency interval: in these cases, when spatial separation is considered, frequency bands are not separated (i.e., dispersion curves of different modes cross and mix), and when a complete frequency gap is occurring between two bands, SW spatial separation is not occurring for all the frequencies of the corresponding bands, and hence there is no duality $[4,28]$. For example, in a typical dot lattice [15], end mode bands are separated by a significant gap from extended wave bands; however, in the upper bands the dynamic magnetization involves even the region of the end modes: a frequency band separation does not strictly meet a SW spatial separation. The same can be said for other kinds of localized SWs in dot or antidot lattices and even bicomponent systems $[4,8,29]$.

\section{Results}

The dynamic magnetization of each mode, resulting as output of DMM calculations, can be interpreted as a Bloch wave, using the following expression:

$$
\delta \mathbf{m}_{k}(\mathbf{r})=\delta \widetilde{\mathbf{m}}_{k}(\mathbf{r}) e^{i \mathbf{k} \cdot \mathbf{r}},
$$

where $\mathbf{r}$ is the position vector in the direct space, $\mathbf{k}$ is the Bloch wavevector $\left(\mathbf{k}_{1}\right.$ or $\mathbf{k}_{2}$, when relevant to the primary or secondary sublattice, correspondingly), and $\delta \widetilde{\mathbf{m}}_{k}$ is the cell function, which has the periodicity of the array. In the following, we will consider only the case of uniform oscillation in the primitive cell (of either of the sublattices) and study the variation of frequency with $k$. Due to the fact that we used the primitive cell of Figure 1 for calculations, we could directly control $k_{2}$ and only get an indirect evaluation of $k_{1}$, at each frequency, after numerical inspection of the mode phase profiles, by extracting the effective wavelength concerning the primary sublattice elements and computing the corresponding wavevector value $\left(k_{1}\right)$.

The dual band property of the system allows the simultaneous propagation of two independent signals, of course, within the linear regime, which in our calculations was used to transform the Landau-Lifshts equation into a linear eigenvalue/eigenvector problem, while in real experiments it can be provided by a proper limitation of the excitation power [8]. The two signals could be excited by either ordinary or polarized current flowing on antennas or point contacts that are preliminary located on one or more elements of either of the lattices [30-33]. Another possibility is an electric 


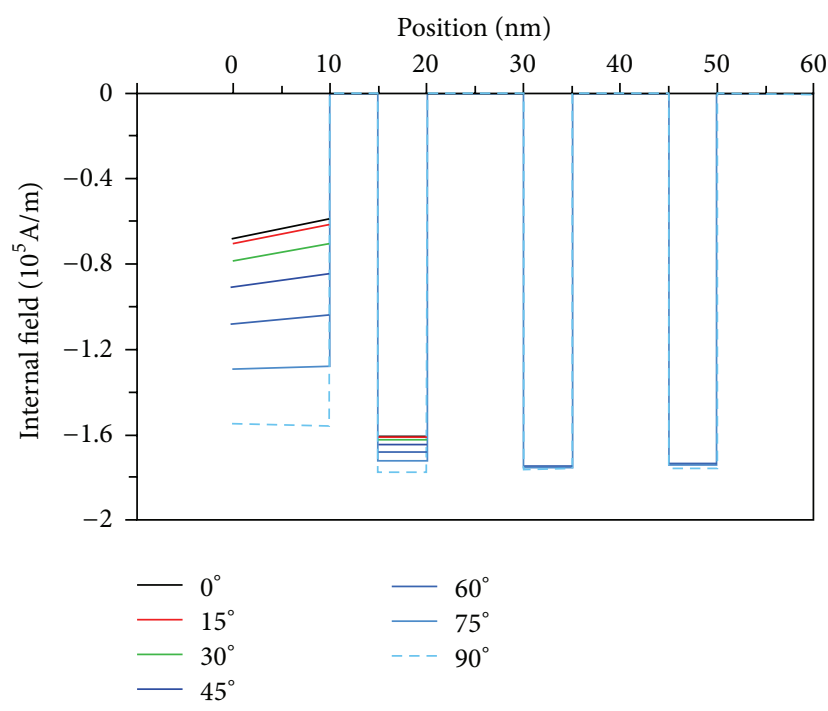

FIGURE 2: Spatial profiles of the internal effective field, for different directions of the applied field of constant magnitude $\mu_{0} H_{0}=0.1 \mathrm{~T}$ (degrees, indicated in figure). Note that the internal field is zero in correspondence with the void spaces. At $0^{\circ}$ the discrepancy between the region $0-10 \mathrm{~nm}$ and the other regions is the largest, while at $90^{\circ}$ it is the minimum.

excitation of a ferroelectric substrate under the MC, by means of the ferroelectric/ferromagnetic interaction $[9,34]$. Recently, even the possibility of exciting a MC by means of optical/microwave frequencies has been explored, resulting in the need for defects in order to get a local excitation and opening the door to studies of defects in MCs [3538]. In this way, we would like to contribute to the research demanding, from one side, ever smaller devices and, from the other side, ever higher density of information, either stored or delivered at frequencies within the $\mathrm{GHz}$ regime: in our model, these two apparently competing features meet in a simple, experimentally challenging answer.

In Figure 2, we plot the internal effective field $H_{\text {eff }}=$ $B_{0} / \mu_{0}+H_{\text {ex }}+H_{\text {dip }}$ (i.e., the sum of the external field $H_{0}=B_{0} / \mu_{0}$ and the exchange $H_{\mathrm{ex}}$ and dipolar $H_{\text {dip }}=$ $-\left|H_{\text {dip }}\right|$ internal fields) as a function of position along $x$ axis (with reference to Figure 1), for different directions of the applied field. From the plot, it is clear that the field direction, $\theta$, critically affects $H_{\text {eff }}$ values only in the bigger elements (secondary sublattice), while variations of $H_{\text {eff }}$ in the smaller elements (primary sublattice) are rather small. Moreover, the mean values in the two sublattices are the farthest at $\theta=0^{\circ}$ and the closest at $\theta=90^{\circ}$ : actually, in the bigger elements, the dipolar field significantly increases as the external field is gradually rotated, because it forces the magnetization to progressively align to the minor axis of the particle (the secondary lattice element), suffering an increasing demagnetizing effect. Since spin wave frequency is critically dependent on the internal effective field, just from this observation it is possible to predict that both the spin waves will occur within either one sublattice or the other, and the corresponding mode frequency difference (magnonic gap) will be the largest at $0^{\circ}$.
In Figure 3 we show the spatial maps of the fundamental modes $[\mathbf{k}=(0,0)]$ that we found: clearly, the dynamic magnetization is localized in either one sublattice or the other, and this property is rigorously hold even for any other wavevector value and direction. We note here that the profile of the fundamental mode relative to the primary sublattice (Figure 3(a)) has a nonuniform amplitude distribution, differently from the secondary one (Figure 3(b)): this is due to the fact that calculations are performed with the primitive cell of Figure 1 (superposition of both sublattices), which is in fact nonunitary for the primary sublattice and contains an element of the secondary sublattice (acting as a sort of "defect" for the primary sublattice, though periodic as well): this fact gives origin to a nonuniform distribution of the dipolar fields among the elements of the primary sublattice (squares) that are contained in the nonunitary primitive cell and, in turn, a nonuniform distribution of the dynamic magnetization of the corresponding quasi-uniform mode, shown in the figure. For this reason, when trying to extract from Figure 3(a) the "ideal" unitary cell function $\delta \widetilde{\mathbf{m}}_{k}(\mathbf{r})$ relevant to the primary sublattice only, it is necessary to forget about the "real" intensity modulation and take the lowest periodic area $\left(a_{1} \times a_{1}\right)$ containing a single square element only (with reference to the red line square of Figure 1), while for the secondary sublattice the "ideal" cell function $\delta \widetilde{\mathbf{m}}_{k}(\mathbf{r})$ can be extracted from Figure 3(b) considering the area $\left(a_{2} \times a_{2}\right)$ corresponding to the extension shown in Figure 1, with a single rectangle and no square elements. However, the realistic cell function $\delta \widetilde{\mathbf{m}}_{k}(\mathbf{r})$, which we calculate and associate to the dual band MC fundamental modes, is the superposition of the two "ideal" ones and can be found in Figures $3(\mathrm{a})$ and $3(\mathrm{~b})$ by simply looking at the area $\left(a_{2} \times a_{2}\right)$ exactly corresponding to what is shown in Figure 1.

Moreover, in Figure 4 we show the magnonic gap for the two modes at $\mathbf{k}=(0,0)$ as a function of the applied field direction: and, as predicted from Figure 2, the gap is continuously decreasing as the applied field direction moves from $0^{\circ}$ to $90^{\circ}$.

In Figure 5 we plot the dispersion curves of the SW modes propagating across either the primary (a) or secondary (b) lattice, when the external field is applied at $\theta=0^{\circ}$. Red and blue lines correspond to propagation perpendicular (with reference to thin film nomenclature, Damon-Eshbach-like) and parallel (backward-like) to the applied field, respectively [39-41]. The former increase as the wavevector magnitude is increased, and saturate, due to the full dipolar nature of the interaction among the lattice elements, in both cases. The latter, instead, decrease with increasing the wavevector magnitude, once more due to the dipolar interaction. From these curves, we compute the average group velocity $v_{g}$ for SWs of either of the sublattices ( $a$ is the proper lattice constant), being

$$
\left\langle v_{g}\right\rangle=\left\langle\frac{d \omega}{d k}\right\rangle=\frac{|\omega(\pi / a)-\omega(0)|}{\pi / a}
$$

which is an indication of the propagation speed of the wave along a given direction. With reference to Figure 5, we calculate different values of $v_{g}$, depending on which lattice 


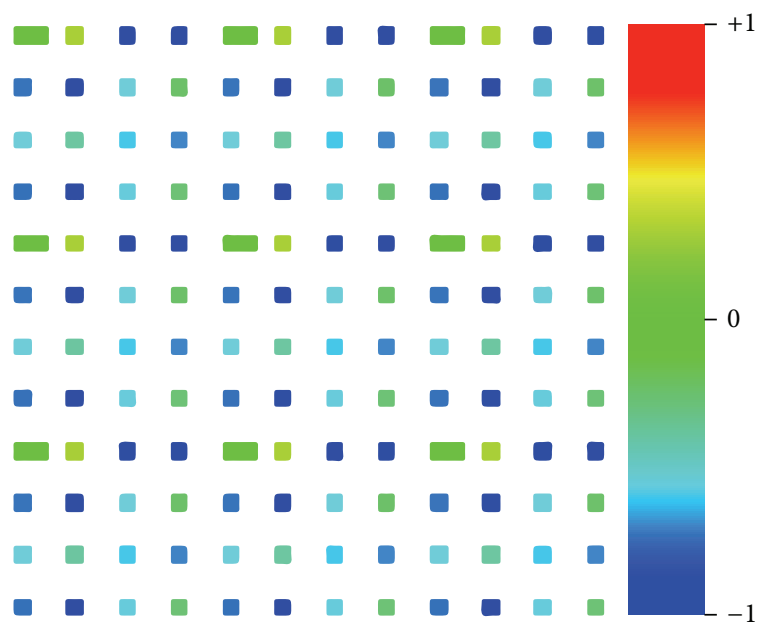

(a)

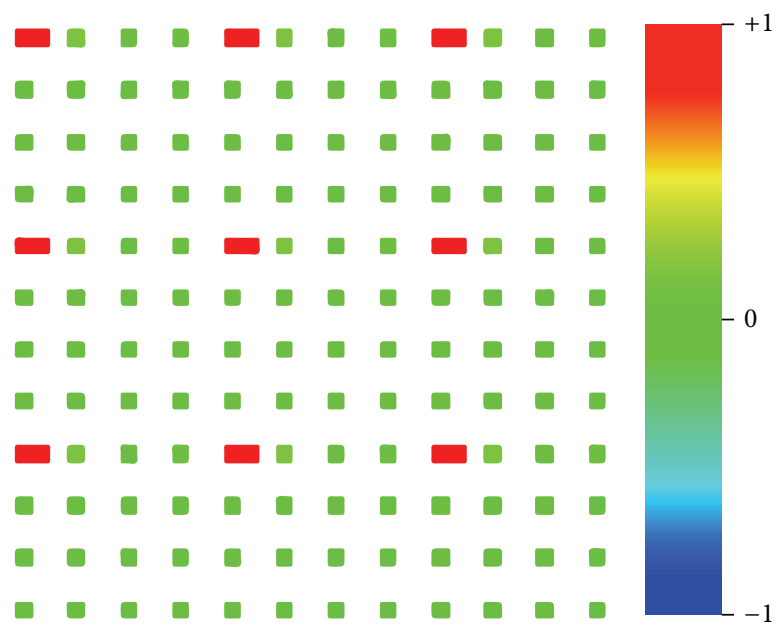

(b)

FIGURE 3: Out-of-plane real component of the dynamic magnetization (arbitrary units) for the two fundamental modes localized in complementary regions of the array (with $\mathbf{B}$ at $\theta=0^{\circ}$ ), consisting of the superposition of two sublattices with different lattice constant. Here, we plot an illustrative $3 \times 3$ periodic cell extract of the ideally infinite $2 \mathrm{D}$ array. (a) Fundamental spin wave mode $\left(k_{1}=0\right)$ localized within the elements of the primary sublattice only, showing an irregular intensity distribution discussed in the text; (b) fundamental spin wave mode $\left(k_{2}=0\right)$ strictly localized in the secondary sublattice.

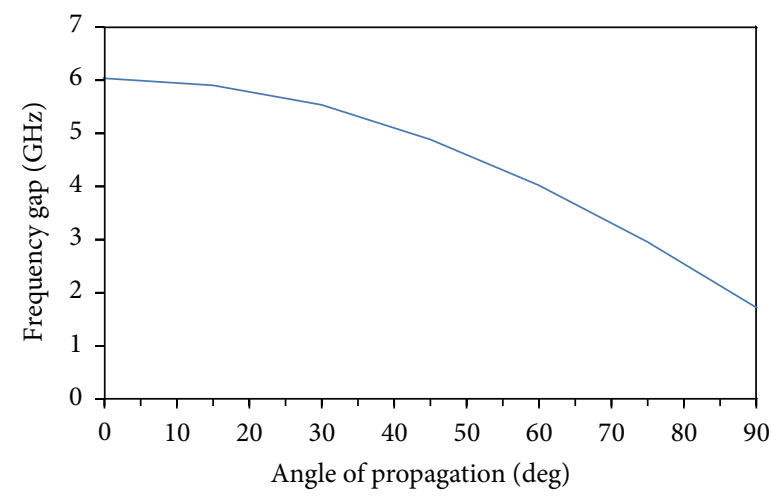

FIGURE 4: Frequency gap between the two spin wave bands as a function of the propagation direction. In this case SW propagation is always parallel to the applied field direction $\theta$ (defined in Figure 1). The gap decreases as a consequence of the frequency decrease of the upper band, due to the increasing of the demagnetizing field in the elongated elements of the lattice (secondary sublattice).

type (primary/secondary) and propagation direction $\mathbf{k}$ with respect to the applied field $\mathbf{B}$ are considered:

$$
\left\langle v_{g}\right\rangle= \begin{cases}2.07 \mathrm{~m} / \mathrm{s} & \text { primary sublattice, } \mathbf{k} \perp \mathbf{B} \\ 11.6 \mathrm{~m} / \mathrm{s} & \text { primary sublattice, } \mathbf{k} \| \mathbf{B} \\ 0.600 \mathrm{~m} / \mathrm{s} & \text { secondary sublattice, } \mathbf{k} \perp \mathbf{B} \\ 1.68 \mathrm{~m} / \mathrm{s} & \text { secondary sublattice, } \mathbf{k} \| \mathbf{B} .\end{cases}
$$

These values are rather low, considering that often SW propagation speed can get the $\mathrm{km} / \mathrm{s}$ order of magnitude: however, the low speed is here a consequence of a low interaction among the MC elements, and, moreover, in $\mathrm{MC}$ devices, due to miniaturization, it is the SW speed difference that is crucial, in order to associate different SWs to different signals or digits. Of course, any other orientation of $\mathbf{k}$ with $\mathbf{B}$ can be, in principle, computed: the above values are shown just to give a test characterization of our choice of MC. Moreover, any other direction $\theta$ of the applied field $\mathbf{B}$ can be considered, which we already know to have little effects on the primary sublattice and major ones on the secondary sublattice.

\section{Comments and Remarks}

The key element in the choice of our system is twofold: from one side, the superposition of two sublattices each made up of nanoparticles with different aspect ratio, that is, with large anisotropy differences, and from the other side, the small (nanometric) size of the lattice constituents. The first aspect (anisotropy) determines the presence of two fundamental modes at $k=0$, localized in different sublattices; each one of these modes can be in principle independently excited, due to the large bandgap. The other aspect (nanometric size) wipes many modes out of the overall low-frequency dynamics, making the occurrence of crossing dispersion curves unlikely: with our choice of taking the micromagnetic cell as large as the nanoparticle (size $5 \mathrm{~nm}$ ), we actually are under the so-called macrospin approximation [42], since when the dot size is lower than the exchange length (in our case, $5.5 \mathrm{~nm}$ ), each nanoparticle acts as a single magnetic moment. With this choice, clearly we will not consider modes with a nonuniform cell function profile [43], that is, modes with nonuniform oscillations within the single nanoparticle, or localized modes (like the end modes in dots with rectangular cross section) [44, 45]: in our system these modes would occur at a very high frequency (close to 


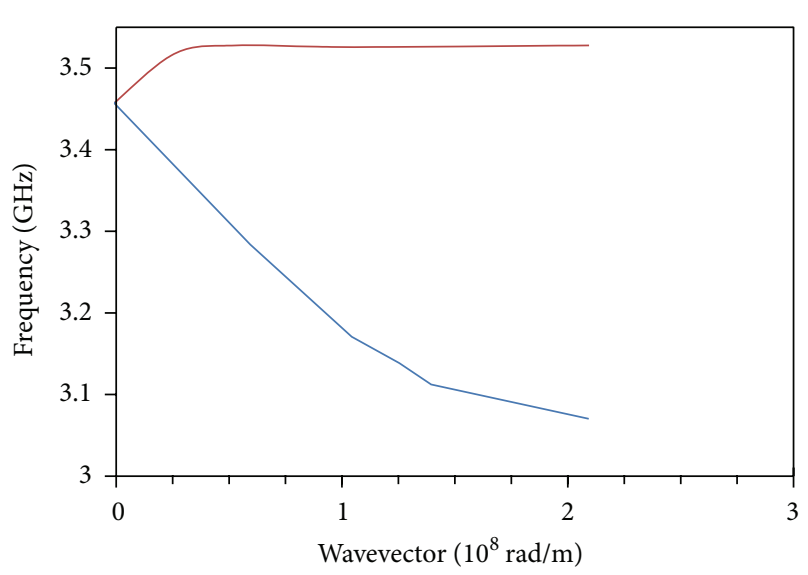

(a)

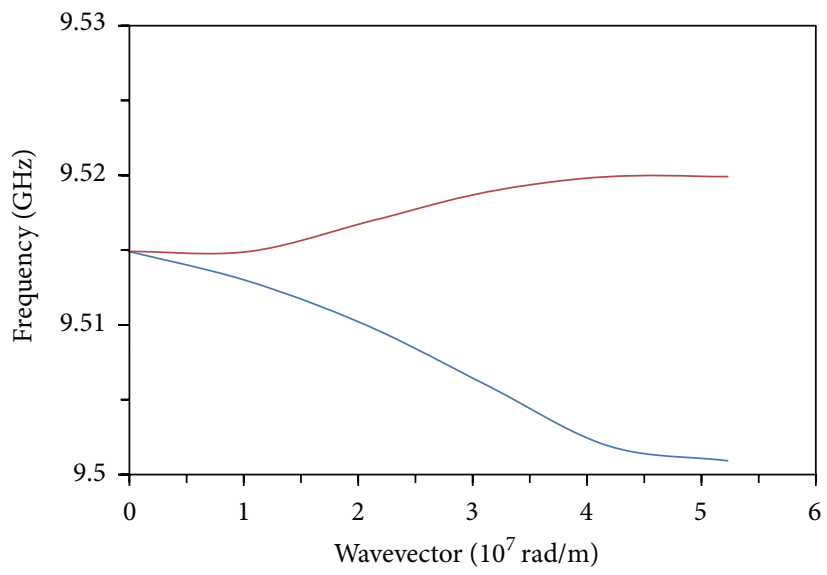

(b)

FIGURE 5: Dispersion relations of SW localized within the primary sublattice ((a), $\left.a_{1}=15 \mathrm{~nm}\right)$, and secondary sublattice ((b), $\left.a_{2}=60 \mathrm{~nm}\right)$; in (a) and (b), the red line corresponds to the DE-like mode $(k \perp B)$ and blue one to the BA-like mode $(k \| B)$. Zone boundary is at $k_{1}=2.09 \times 10^{8} \mathrm{rad} / \mathrm{m}$ (a) and $k_{2}=5.23 \times 10^{7} \mathrm{rad} / \mathrm{m}(\mathrm{b})$. Note that in (b) the wavevector $k_{2}$ was a parameter of the calculation, while in (a) $k_{1}$ was extrapolated by phase map inspection.

or larger than $100 \mathrm{GHz})$, so with our choice we do not miss any realistic (low-frequency) solution.

Concerning the dual band feature, anisotropy differences at this small length scale are to some extent more important than the filling fraction of the system, that is, the ratio between the actual magnetic area in the periodic cell and the total area of the periodic cell itself. The filling fraction, actually, has proven to be important in determining the opening of a complete magnonic bandgap in magnonic crystals $[46,47]$. Within a micromagnetic model, we can increase the filling fraction by keeping the lattice elements closer to each other, and this would increase the interdot interaction and decrease the internal field differences between the elements of each lattice: as a direct consequence, nanodots belonging to different sublattices would be less independent, so that excitations of one sublattice would interfere with the other one, breaking the dual property. Conversely, if we decrease the filling factor by keeping the lattice elements far apart, the system would behave more and more as a set of degenerate (noninteracting) dots, with very flat dispersion curves, less attractive for information delivery issues.

On the other side, for any given filling fraction, different systems characterized by increasing dot size (larger than the exchange length) would more and more tend to break the macrospin picture, due to the increasing rise of modes with complex (nonuniform) cell function, with increasing chances of crossing among different mode dispersions, so that the frequency bandgap between the fundamental modes of the two sublattices can be extremely reduced or not present at all; moreover, due to the consequent increasing interdot distance, the interdot interaction would be lower, with resulting flatter dispersion curves, with smaller group velocities.

A remarkable feature of truly dual band MCs is that it is not possible to interpret the long wavelength behavior $(k=0)$ of the SW dynamics within the usual effective medium model [48-51]: this model approximates the system by a single effective medium with effective (intermediate) properties, with a resulting single effective frequency at $k=0$. But the existence of a very large fluctuation of the internal field values between the two different sublattices in the real system (at the origin of the existence of two fundamentallike SW modes with a very large frequency gap even at $k=$ 0 ) makes any intermediate ("effective") value a nonphysical solution. Similar situations were discussed in other systems, with similar large fluctuations in the internal field [52-54]: in particular, in dots with triangular cross section [52] it was shown that an effective Kittel (quasi-uniform) mode was impossible to consider as an actual solution, because of the very strong variation of the internal field from the apex to the basis of the triangle.

\section{Conclusion}

In this paper, we proposed and characterized a model system of MC, in which SWs occur in two bands, separated by a very large frequency gap. SWs of either of the bands propagate in different regions of the MC, with no superposition, and can be considered completely independent. This allows considering the system as ideal for simultaneous propagation of two independent signals, at very different frequencies. This property, which was designed by exploiting different shape anisotropy of different MC elements, is of great importance in technology of miniaturized devices for high-density information storage/delivery, where, usually, the need for miniaturization contrasts with the other important need of signal independence, due to magnetic interactions. This work is intended to be introductory for further investigations, both theoretical and experimental, on combined systems, made of superposed lattices with special design, geometry and, possibly, different magnetic materials, all tailored in order to get a very sharp change of the internal field profile across the medium: this feature was found to be the key ingredient for the occurrence of dual band SW dynamics. 


\section{Competing Interests}

The authors declare that they have no competing interests.

\section{References}

[1] R. L. Stamps, S. Breitkreutz, J. Åkerman et al., "The 2014 magnetism roadmap," Journal of Physics D: Applied Physics, vol. 47, no. 33, Article ID 333001, 2014.

[2] M. Krawczyk and D. Grundler, "Review and prospects of magnonic crystals and devices with reprogrammable band structure," Journal of Physics: Condensed Matter, vol. 26, no. 12, Article ID 123202, 2014.

[3] G. Gubbiotti, F. Montoncello, S. Tacchi et al., "Angle-resolved spin wave band diagrams of square antidot lattices studied by Brillouin light scattering," Applied Physics Letters, vol. 106, no. 26, Article ID 262406, 2015.

[4] M. Krawczyk, S. Mamica, M. Mruczkiewicz et al., "Magnonic band structures in two-dimensional bi-component magnonic crystals with in-plane magnetization," Journal of Physics D: Applied Physics, vol. 46, no. 49, Article ID 495003, 2013.

[5] S. Mamica, "Tailoring of the partial magnonic gap in threedimensional magnetoferritin-based magnonic crystals," Journal of Applied Physics, vol. 114, no. 4, Article ID 043912, 2013.

[6] J. Topp, D. Heitmann, M. P. Kostylev, and D. Grundler, "Making a reconfigurable artificial crystal by ordering bistable magnetic nanowires," Physical Review Letters, vol. 104, no. 20, Article ID 207205, 2010.

[7] H. Yu, G. Duerr, R. Huber et al., "Omnidirectional spin-wave nanograting coupler," Nature Communications, vol. 4, article 2702, 2013.

[8] B. Van de Wiele and F. Montoncello, "A continuous excitation approach to determine time-dependent dispersion diagrams in 2D magnonic crystals," Journal of Physics D: Applied Physics, vol. 47, no. 31, p. 315002, 2014.

[9] B. Van de Wiele, S. J. Hämäläinen, P. Baláž, F. Montoncello, and S. van Dijken, "Tunable short-wavelength spin wave excitation from pinned magnetic domain walls," Scientific Reports, vol. 6, Article ID 21330, 2016.

[10] S. Klingler, P. Pirro, T. Brächer, B. Leven, B. Hillebrands, and A. V. Chumak, "Design of a spin-wave majority gate employing mode selection," Applied Physics Letters, vol. 105, no. 15, Article ID 152410, 2014.

[11] S.-K. Kim, K.-S. Lee, and D.-S. Han, "A gigahertz-range spinwave filter composed of width-modulated nanostrip magnoniccrystal waveguides," Applied Physics Letters, vol. 95, no. 8, Article ID 082507, 2009.

[12] A. V. Chumak, V. I. Vasyuchka, A. A. Serga, M. P. Kostylev, V. S. Tiberkevich, and B. Hillebrands, "Storage-recovery phenomenon in magnonic crystal," Physical Review Letters, vol. 108, no. 25, Article ID 257207, 2012.

[13] A. A. Serga, A. V. Chumak, A. André et al., "Parametrically stimulated recovery of a microwave signal stored in standing spin-wave modes of a pagnetic film," Physical Review Letters, vol. 99, no. 22, Article ID 227202, 2007.

[14] F. Montoncello and L. Giovannini, "Bandwidth broadening and asymmetric softening of collective spin waves in magnonic crystals," Applied Physics Letters, vol. 104, no. 24, Article ID 242407, 2014.

[15] F. Montoncello, S. Tacchi, L. Giovannini et al., "Asymmetry of spin wave dispersions in a hexagonal magnonic crystal," Applied Physics Letters, vol. 102, no. 20, Article ID 202411, 2013.
[16] F. Montoncello and L. Giovannini, "Vortex mode dynamics and bandwidth tunability in a two-dimensional array of interacting magnetic disks," Applied Physics Letters, vol. 100, no. 18, Article ID 182406, 2012.

[17] A. V. Khvalkovskiy, D. Apalkov, S. Watts et al., "Erratum: basic principles of STT-MRAM cell operation in memory arrays," Journal of Physics D: Applied Physics, vol. 46, no. 13, Article ID 139601, 2013.

[18] A. V. Chumak, A. A. Serga, and B. Hillebrands, "Magnon transistor for all-magnon data processing," Nature Communications, vol. 5, article 4700, 2014.

[19] A. Khitun, M. Bao, and K. L. Wang, "Magnonic logic circuits," Journal of Physics D: Applied Physics, vol. 43, no. 26, Article ID 264005, 2010.

[20] A. V. Chumak, V. I. Vasyuchka, A. A. Serga, and B. Hillebrands, "Magnon spintronics," Nature Physics, vol. 11, no. 6, pp. 453-461, 2015.

[21] L. J. Heyderman and R. L. Stamps, "Artificial ferroic systems: novel functionality from structure, interactions and dynamics," Journal of Physics Condensed Matter, vol. 25, no. 36, Article ID 363201, 2013.

[22] M. J. Donahue and D. G. Porter, OOMMF User's Guide, Version 1.0, NIST, Gaithersburg, Md, USA, 1999.

[23] L. Giovannini, F. Montoncello, and F. Nizzoli, "Effect of interdot coupling on spin-wave modes in nanoparticle arrays," Physical Review B, vol. 75, no. 2, Article ID 024416, 2007.

[24] O. Kasyutich, R. D. Desautels, B. W. Southern, and J. Van Lierop, "Novel aspects of magnetic interactions in a macroscopic 3D nanoparticle-based crystal," Physical Review Letters, vol. 104, no. 12, Article ID 127205, 2010.

[25] O. Kasyutich, D. Tatchev, A. Hoell, F. Ogrin, C. Dewhurst, and W. Schwarzacher, "Small angle X-ray and neutron scattering study of disordered and three dimensional-ordered magnetic protein arrays," Journal of Applied Physics, vol. 105, no. 7, Article ID 07B528, 2009.

[26] O. Kasyutich, A. Sarua, and W. Schwarzacher, "Bioengineered magnetic crystals," Journal of Physics D: Applied Physics, vol. 41, no. 13, Article ID 134022, 2008.

[27] F. Montoncello, L. Giovannini, and M. Krawczyk, "Spin wave localization and softening in rod-shaped magnonic crystals with different terminations," Journal of Applied Physics, vol. 112, no. 3, Article ID 033911, 2012.

[28] S. Tacchi, P. Gruszecki, M. Madami et al., "Universal dependence of the spin wave band structure on the geometrical characteristics of two-dimensional magnonic crystals," Scientific Reports, vol. 5, Article ID 10367, 2015.

[29] R. Zivieri, S. Tacchi, F. Montoncello et al., "Bragg diffraction of spin waves from a two-dimensional antidot lattice," Physical Review B, vol. 85, no. 1, Article ID 012403, 2012.

[30] A. V. Chumak, A. A. Serga, S. Wolff, B. Hillebrands, and M. P. Kostylev, "Scattering of surface and volume spin waves in a magnonic crystal," Applied Physics Letters, vol. 94, no. 17, Article ID 172511, 2009.

[31] A. V. Chumak, A. A. Serga, S. Wolff, B. Hillebrands, and M. P. Kostylev, "Design and optimization of one-dimensional ferritefilm based magnonic crystals," Journal of Applied Physics, vol. 105, no. 8, Article ID 083906, 2009.

[32] M. Madami, S. Bonetti, G. Consolo et al., "Direct observation of a propagating spin wave induced by spin-transfer torque," Nature Nanotechnology, vol. 6, no. 10, pp. 635-638, 2010. 
[33] V. E. Demidov, S. Urazhdin, H. Ulrichs et al., "Magnetic nanooscillator driven by pure spin current," Nature Materials, vol. 11, no. 12, pp. 1028-1031, 2012.

[34] B. Van De Wiele, L. Laurson, K. J. A. Franke, and S. Van Dijken, "Electric field driven magnetic domain wall motion in ferromagnetic- ferroelectric heterostructures," Applied Physics Letters, vol. 104, no. 1, Article ID 012401, 2014.

[35] Y. Au, E. Ahmad, O. Dmytriiev, M. Dvornik, T. Davison, and V. V. Kruglyak, "Resonant microwave-to-spin-wave transducer," Applied Physics Letters, vol. 100, no. 18, Article ID 182404, 2012.

[36] B. Koene, M. Savoini, A. V. Kimel, A. Kirilyuk, and T. Rasing, "Optical energy optimization at the nanoscale by near-field interference," Applied Physics Letters, vol. 101, no. 1, Article ID 013115, 2012.

[37] J. Ding, M. Kostylev, and A. O. Adeyeye, "Magnonic crystal as a medium with tunable disorder on a periodical lattice," Physical Review Letters, vol. 107, no. 4, Article ID 047205, 2011.

[38] N. Ross, M. Kostylev, and R. L. Stamps, "Effect of disorder studied with ferromagnetic resonance for arrays of tangentially magnetized submicron Permalloy disks fabricated by nanosphere lithography," Journal of Applied Physics, vol. 109, no. 1, Article ID 013906, 2011.

[39] R. W. Damon and J. R. Eshbach, "Magnetostatic modes of a ferromagnet slab," Journal of Physics and Chemistry of Solids, vol. 19, no. 3-4, pp. 308-320, 1961.

[40] R. M. White, Quantum Theory of Magnetism: Magnetic Properties of Materials, chapter 8, Springer, Berlin, Germany, 2007.

[41] G. Venkat, D. Kumar, M. Franchin et al., "Proposal for a standard micromagnetic problem: spin wave dispersion in a magnonic waveguide," IEEE Transactions on Magnetics, vol. 49, no. 1, pp. 524-529, 2013.

[42] D. V. Berkov and J. Miltat, "Spin-torque driven magnetization dynamics: micromagnetic modeling," Journal of Magnetism and Magnetic Materials, vol. 320, no. 7, pp. 1238-1259, 2008.

[43] C. Bayer, M. P. Kostylev, and B. Hillebrands, "Spin-wave eigenmodes of an infinite thin film with periodically modulated exchange bias field," Applied Physics Letters, vol. 88, no. 11, Article ID 112504, 2006.

[44] H. Puszkarski, M. Krawczyk, and J.-C. S. Lévy, "Localization properties of pure magnetostatic modes in a cubic nanograin," Physical Review B, vol. 71, no. 1, Article ID 014421, 2005.

[45] M. Grimsditch, G. K. Leaf, H. G. Kaper, D. A. Karpeev, and R. E. Camley, "Normal modes of spin excitations in magnetic nanoparticles," Physical Review B, vol. 69, no. 17, Article ID 174428, 2004.

[46] M. Krawczyk and H. Puszkarski, "Plane-wave theory of threedimensional magnonic crystals," Physical Review B, vol. 77, no. 5, Article ID 054437, 13 pages, 2008.

[47] S. Mamica, M. Krawczyk, M. L. Sokolovskyy, and J. RomeroVivas, "Large magnonic band gaps and spectra evolution in three-dimensional magnonic crystals based on magnetoferritin nanoparticles," Physical Review B, vol. 86, no. 14, Article ID 144402, 2012.

[48] N. S. Almeida and D. L. Mills, "Effective-medium theory of long-wavelength spin waves in magnetic superlattices," Physical Review B, vol. 38, no. 10, pp. 6698-6710, 1988.

[49] X.-Z. Wang and D. R. Tilley, "Magnetostatic modes on lateral magnetic superlattices," Physics Letters A, vol. 187, no. 4, pp. 325330, 1994

[50] X.-Z. Wang and D. R. Tilley, "Magnetostatic surface and guided modes of lateral-magnetic-superlattice films," Physical Review B, vol. 50, no. 18, pp. 13472-13479, 1994.
[51] X.-Z. Wang and D. R. Tilley, "Magnetostatic modes of lateralmagnetic-superlattice films in a transverse field," Journal of Physics: Condensed Matter, vol. 9, no. 27, pp. 5777-5786, 1997.

[52] F. Montoncello and F. Nizzoli, "Spin modes of triangular magnetic nanodots in the vortex, Y, and buckle states," Journal of Applied Physics, vol. 107, no. 2, Article ID 023906, 2010.

[53] F. Montoncello, L. Giovannini, F. Nizzoli, P. Vavassori, and M. Grimsditch, "Dynamic origin of first and second order phase transitions in magnetization reversal of elliptical nanodots," Physical Review B, vol. 77, no. 21, Article ID 214402, 2008.

[54] F. Montoncello, L. Giovannini, F. Nizzoli et al., "Magnetization reversal and soft modes in nanorings: transitions between onion and vortex states studied by Brillouin light scattering," Physical Review B, vol. 78, no. 10, Article ID 104421, 7 pages, 2008. 

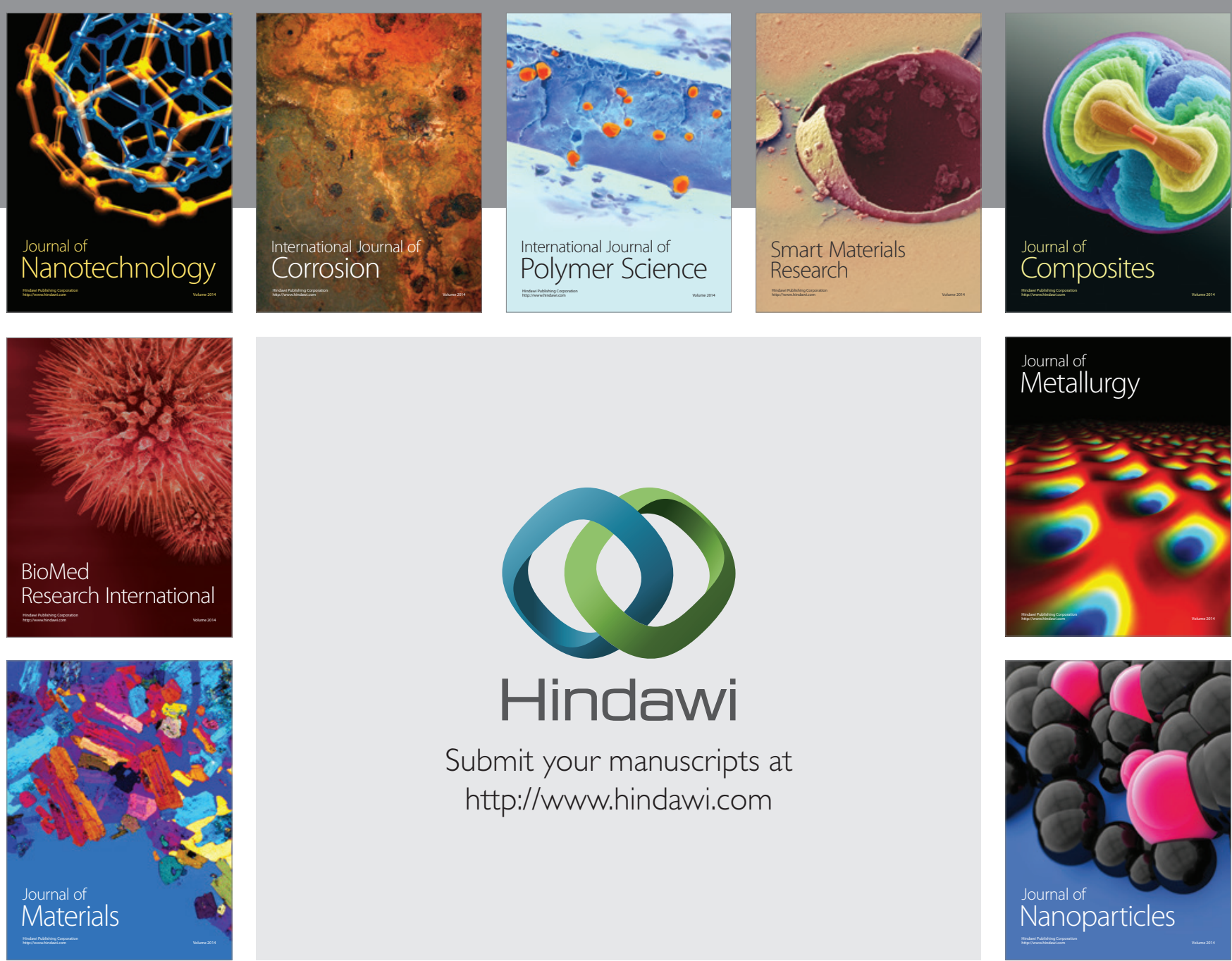

\section{Hindawi}

Submit your manuscripts at

http://www.hindawi.com

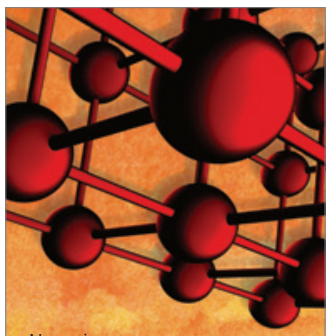

Materials Science and Engineering
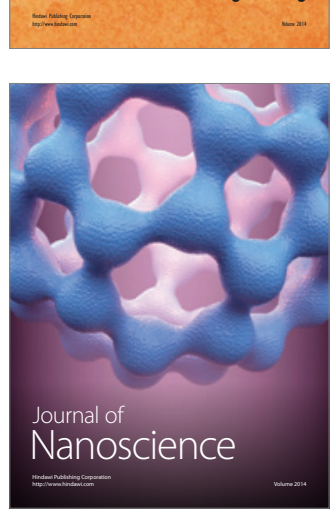
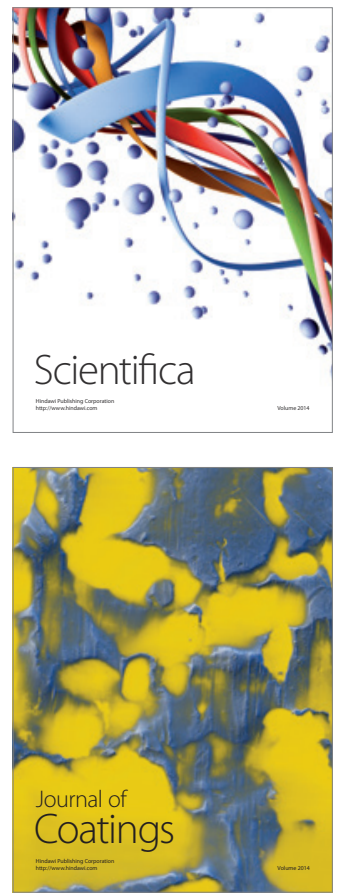
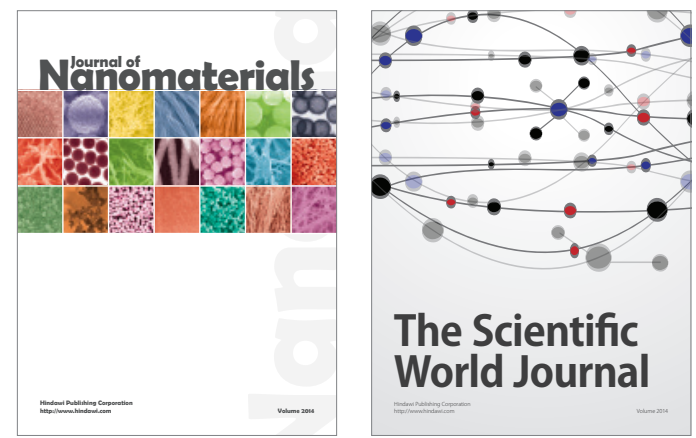

The Scientific World Journal
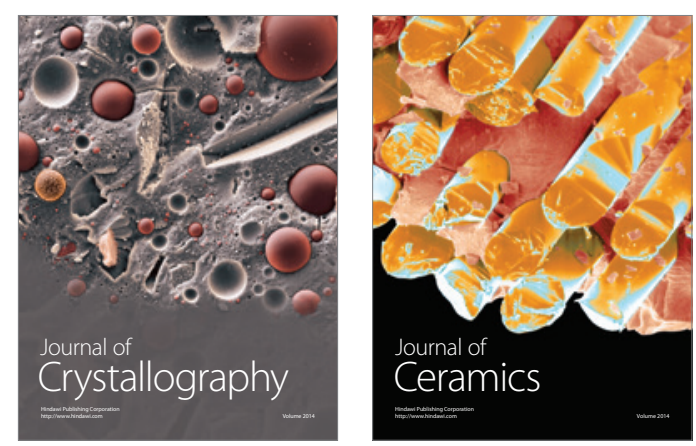
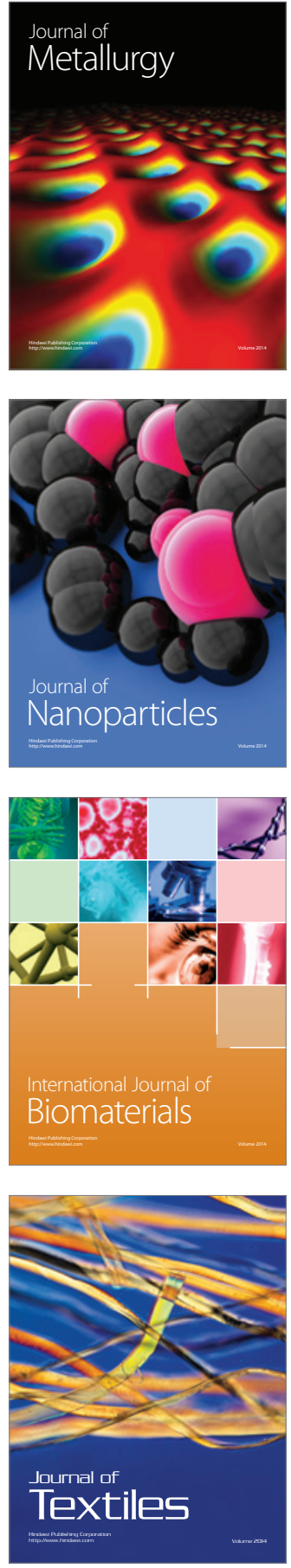